\title{
The Recognition Algorithm of VSAT Network Based on Burst Synchronization
}

\author{
Hairui Pan ${ }^{1, a}$, Yourong $\mathrm{Lu}^{1, \mathrm{~b}}$, Qian ${ }^{1, \mathrm{c}}$ Cai and GangXu ${ }^{1, \mathrm{~d}}$ \\ ${ }^{1}$ Southwest Electronics and Telecommunication Technology Research Institute,Chendu,610041, \\ China;

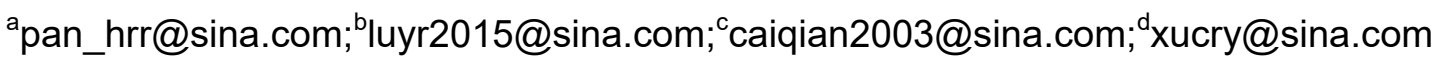

Keywords: unique code; identification of network; slot; TDMA.

\begin{abstract}
For the problem about identification of VSAT network, a VSAT network identification technology using the priori information of VSAT network is proposed based on synchronization among different carriers' bursts. The validation via practical data shows that the method is applicable to the VSAT network of which bursts' length are fixed. It can be applied to networks which have the feature that bursts are synchronized among different carriers. furthermore, it makes a good foundation for the subsequent application. The significance of this paper is that by the detection of unique code and parametric analysis will increase the reliability of results.
\end{abstract}

\section{Introduction}

In this paper, the identification problem of VSAT network, namely finding the carriers' set those belong to the same VSAT satellite communications network (referred to as the VSAT network), will lay a foundation for the subsequent signal and information processing. Currently recognition about VSAT network are few reports in academic. After analyzing and summarizing we found that features about each type of TDMA communication device such as modulation, burst period, distribution of channel, preamble length, and a channel pattern are different, so the characteristics can be used as a basis for identification of VSAT network. Currently unique code detection which is a mature technology can be used to identify the carriers in a VSAT network, but strictly speaking, the carriers detection by unique code can only determine whether they are from the same type of device those have the same unique code or not. In practice, there are a plurality of user groups with a communication device (unique code) and they are different communication networks. This paper argues that different VSAT network using the same type of equipment have different resource allocation types between each other, bursts from different network are not synchronized. For these reasons, we use the feature that bursts from the same network are synchronized to achieve network identification.

In 1953 Baker[1] proposed the correlation-threshold detection method, which use local unique code doing correlation detection with signal. It's simple and easy to implement. In 1972 Massey[2] first proposed the maximum likelihood detection method and its performance are fair with correlation-threshold detection, but it did not consider the effects of frequency offset. Considering offset and phase deviation, Choi[3] derived maximum likelihood detection by fourth-order Bessel expansion, double correlation detection was proposed. The algorithm can inhibit noise and offset effectively. For sampled signal those with undetermined time, Huang Yuan Ling[4] proposed an average likelihood ratio detection algorithm, the method has better robustness for timing error and frequency offset.

\section{Model}

\subsection{VSAT network model}

Referencing [5] and combining with actual signal of VSAT network, This section gives channel resource allocation model (Fig.1) those length of bursts are fixed. From Fig.1 we know bursts from different carriers which are in a same VSAT network are alignment. 


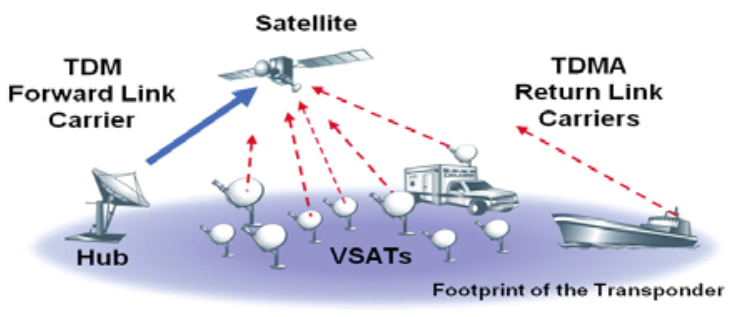

(a)

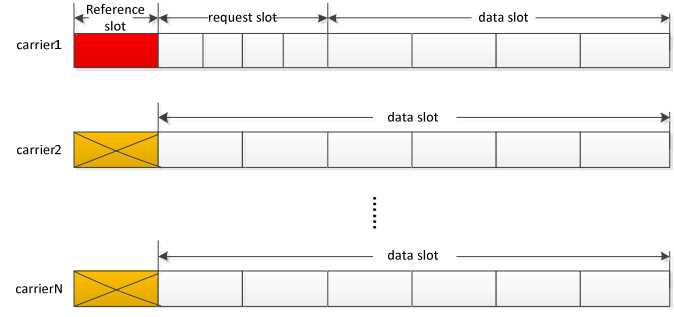

(b)

\section{2 signal model}

Fig. 1 channel resource allocation model

Considering additive Gaussian noise (AWGN),modulation is MPSK. TDMA frame structure is shown in Fig.2. Each frame consists of M slots, each time slot comprises a guard time, a unique code, data, etc. The unique code is fixed with L symbols, the remaining NL data are random symbols (each symbol value equal probability).

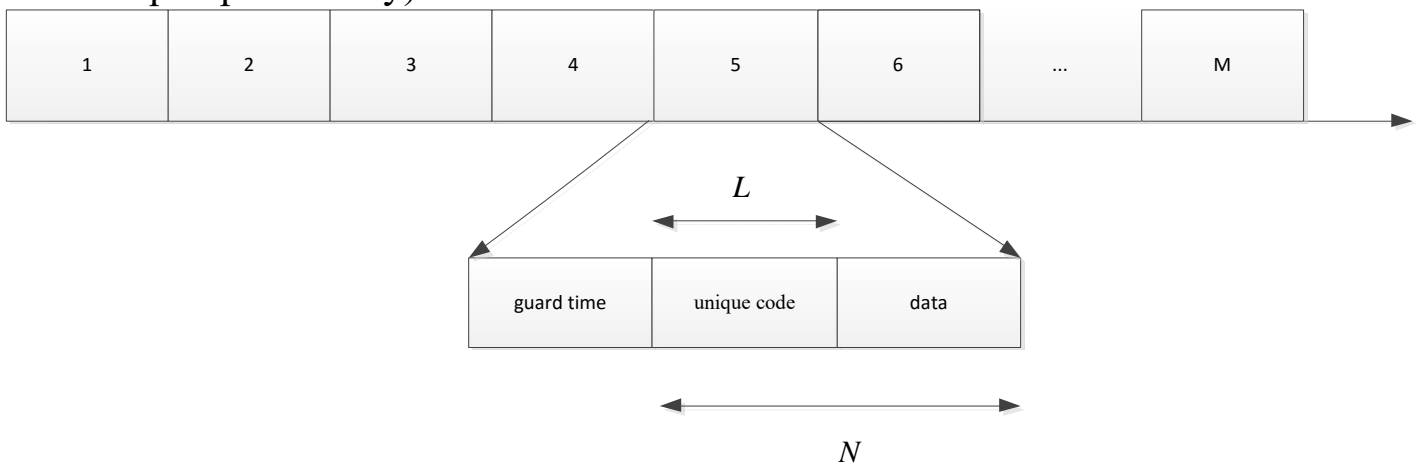

Fig. 2 TDMA frame structure

Based on the above assumptions, signal reception model is as follows[6]:

$$
r_{n}=s_{n} \exp \left(j\left(2 \pi \Delta f \cdot n T_{s}+\phi_{0}\right)\right)+z_{n}, n=1, \ldots, P N
$$

Where $s_{n}=\sum_{k=-\infty}^{\infty} \exp \left(j \varphi_{k}\right) h\left(n T_{s}-k T-\tau\right)$ is a baseband signal. $\left\{\varphi_{k}\right\}$ is phase information of data symbols. $h(t)$ is raised cosine filter. $\tau$ is timing error. $\Delta f$ is Frequency offset. $\phi_{0}$ is phase error, $T_{s}$ is sampling period, $T=P T_{s}$ is symbol period. Supposing that $2 \pi \Delta f \cdot P T_{s}$ and $\phi_{0}$ are subject to the uniform distribution $[-\pi, \pi] . z_{n}$ is additive white Gaussian noise, its mean is 0 and variance is $\sigma^{2}$.

\section{$3 \quad$ Algorithmic Process}

1) Calculating spectrum of MF-TDMA signals for detecting;

2) Separately, estimating frequency, rate and modulation for each carrier estimation;

3) According to frequency, modulation and rate estimated by 2), doing DDC for each carrier, we obtain baseband signals $r_{n}$;

4)Estimating peak period by detection of unique code and got bursts moment $t_{i, f_{k 1}}, t_{j, f_{k 2}}$, if test fails, using other specific unique code those are known (modulation scheme are the same with $r_{n}$ ) to continue detection.

5) Certifying bursts on different carriers are from the same VSAT network or not.

a) $t_{\text {initial }}=\min \left(t_{i, f_{k 1}}, t_{j, f_{k 2}}\right)$;

b) Selecting carrier which modulation rate is high its bursts' moment is $t_{i, f_{k 1}}$;

c) For $\left|n T+t_{\text {initial }}-t_{i, f_{k 1}}\right| \leq \Delta_{1}$, if $n$ makes the inequality establishment permanently, then the test carriers and the reference carrier are synchronized, otherwise they are not synchronized. judgment formula of Double correlation detection is as follows: 


$$
L(\mu)=\sum_{k=1}^{P L-1}\left\{\left|\sum_{i=k}^{P L-1} r_{\mu+i} c_{i}^{*} r_{\mu+i-k}^{*} C_{i-k}\right|^{2}-\sum_{i=\mu+k}^{\mu+P L-1}\left|r_{i}\right|^{2}\left|r_{i-k}\right|^{2}\right\}
$$

The average likelihood ratio estimation result is expressed as

$$
\mu=\underset{\mu}{\arg \max }\left\{\sum_{i=1}^{P L-1}\left|\sum_{k=i}^{P L-1} r_{k}{ }^{*} C_{k} r_{k-i} C_{k-i}{ }^{*}\right|^{2} / \sum_{i=1}^{P L-1} \sum_{k=i}^{P L-1}\left|r_{k} r_{k-i}\right|^{2}\right\}
$$

Where $\mu$ is starting position of unique code, $c_{k}$ is a known unique code.

This paper's work is focused on the step 3) to 5).

\section{$4 \quad$ Verification of Actual Data}

We choose signal with carrier $f_{1}, f_{2}, f_{3}$ and they have the same unique code the bandwidth ratio of them is $1: 2.59$ : 1 , length of signal $\mathrm{T}=0.0107 \mathrm{~s}$. The oversampling is 8 , so that each point represents $1 / 8$ symbol period.

Fig.3(a) and Fig.3(b) are tests by double correlation and average likelihood ratio with the same carrier. Where peaks' location are starting position of unique code. There are two unique code detection method we used so that will increase credibility of parameters estimated, on the other hand is that we can choose a better algorithm which has a better performance. We randomly select three bursts in Fig.3(a) and three corresponding bursts in Fig.3(b).It increases reliability of bursts' moment; bursts' moment can not be accurately determined in Figure 4 because of the actual sampling timing error, while average likelihood ratio test does not exist this phenomenon for its robustness. So average likelihood ratio is better than double correlation slightly.

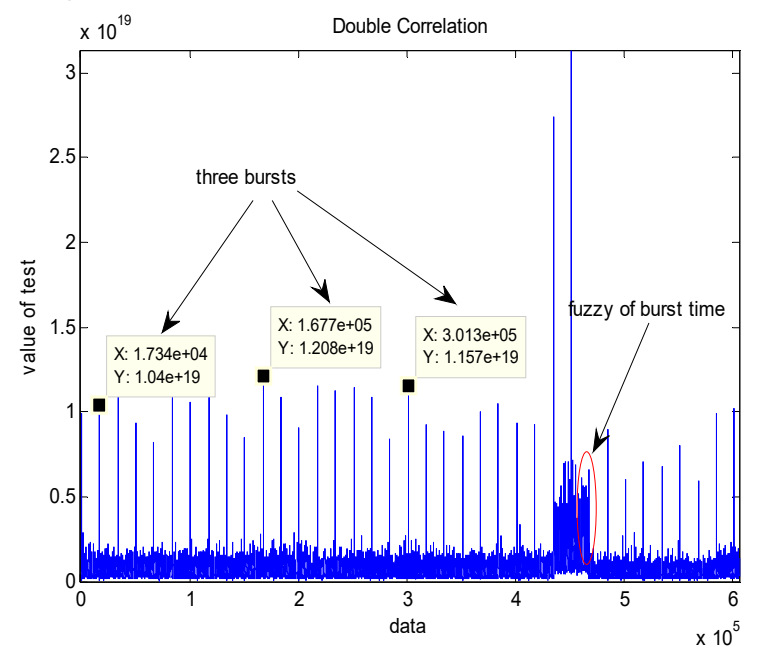

(a)double correlation

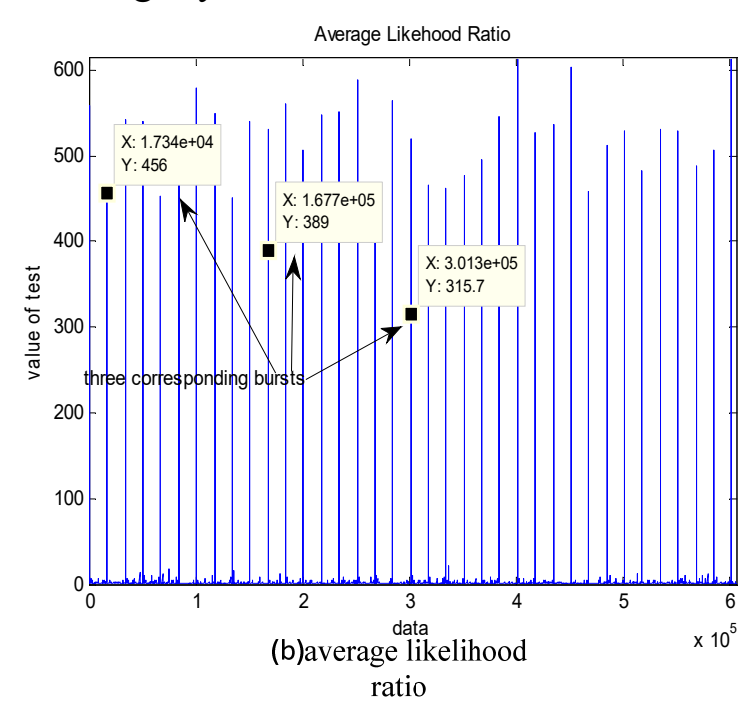

ratio

Fig. 2 double correlation and average likelihood ratio

Fig 4 is bursts' moment diagram of carriers.

Via test, modulation of the three carriers are QPSK and their unique word, length of burst are the same. After analyzing, we know that carrier $f_{1}$ and carrier $f_{3}$ meet the synchronization condition. While trap error $\Delta_{\min }=0.0001 \mathrm{~s}$, carrier $f_{1}$, carrier $f_{2}$ and carrier $f_{3}$ are synchronized. So carrier $f_{1}$ and carrier $f_{3}$ belong to a same network, carrier 1, carrier 2, carrier 3 is likely to belong to a same network.

\section{Summary}

We research identification of VSAT network from the view of signal layer, using the feature that different carriers in the VSAT network are synchronized. A method based on unique code detection is proposed on identification of VSAT network. This method is simple in principle and it is suitable for VSAT network those length of slot are fixed. To some extent, The method proposed can identify carriers from the same VSAT network. 


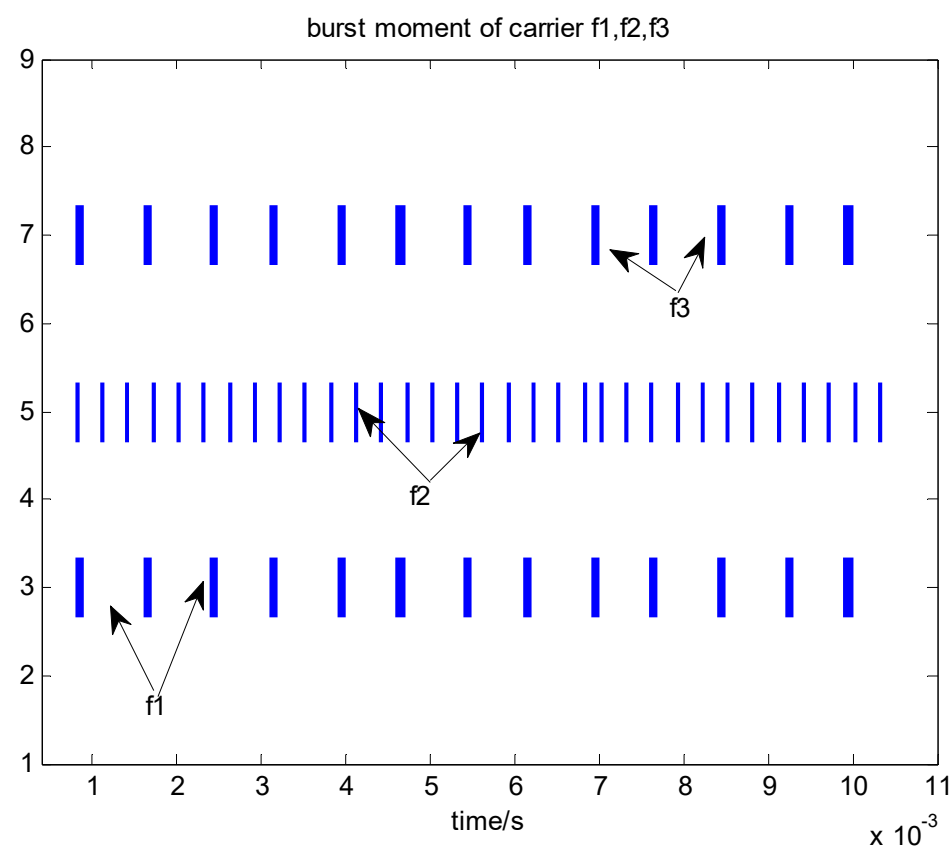

Fig. 3 moment of bursts

\section{References}

[1] H, Barker. Group synchronization of binary digital systems in communication theory, Wackson, Ed.London.Butterworth,1 953,PP.273-287.

[2] J. L. Massey, Optimum frame synchronization, IEEE Trans.Commun, vol. COM-20, pp. 115-119, Apr. 1972.

[3] Zae Yong Choi, Yong Hoon Lee. On the Use of Double Correlation for Frame SynchrOnizatiOn in the Presence of Frequency Offset [C], Proc. IEEE International Conference on Communications (ICC 1999), IEEE Press, June 1999, vo1.2, PP.958-962. doi: 10.1109 / ICC. 1999.765415.

[4] Huang Yuan-ling, Lu You-rong, and Yuan Qiang. Robust Burst Detection Based on the Average Likelihood Ratio Test [J]. Journal of Electronics \& Information Technology, 2010,32 (2): 345-349.

[5] ETSI.Digital Video Broadcasting (DVB), Interaction channel for satellite distribution systems [EB / OL]. (2009-05-01) [2012-10-10]. Http://pda.etsi.org/pda /queryform.asp.

[6] Fenglin Tan, Quan Yang, Ning Wu. Joint Synchronization for Burst Communications Based On Unique Words [C] Wireless Communications Networking and Mobile Computing (WiCOM), 2010 6th International Conference, IEEE Press, 23-25Sept.2010, PP.1-4, doi: 10.1109 / WICOM.2010.5600932. 